\title{
Detection of cocaine induced rat brain activation by photoacoustic tomography
}

\author{
Janggun Jo and Xinmai Yang \\ KU Bioengineering Research Center, Department of Mechanical Engineering, The University of \\ Kansas, 5109 Learned Hall 1530 West 15th Street Lawrence, KS 66045 Tel. (785) 864-1744 Fax. \\ (785) 864-1742 xmyang@ku.edu
}

\begin{abstract}
Photoacoustic tomography (PAT) was used to detect the progressive changes on the cerebral cortex of Sprague Dawley rats after the administration of cocaine hydrochloride. Different concentrations $(0,2.5$, and $5.0 \mathrm{mg}$ per kg body) of cocaine hydrochloride in saline solution were injected into Sprague Dawley rats through tail veins. Cerebral cortex images of the animals were continuously acquired by PAT. For continuous observation, PAT system used multi-transducers to reduce the scanning time and maintain a good signal-to-noise ratio (SNR). The obtained photoacoustic images were compared with each other and confirmed that changes in blood volume were induced by cocaine hydrochloride injection. The results demonstrate that PAT may be used to detect the effects of drug abuse-induced brain activation.
\end{abstract}

\section{Keywords}

Photoacoustic tomography; imaging; Cocaine; drug abuse; brain; cerebral cortex

\section{Introduction}

Chronic drug abuse and addiction cause serious harm to the society and the addicted individuals (Andlin-Sobocki and Rehm, 2005; Grant and Dawson, 1998). The adaptations that occur in brain structure and function caused by chronic drug use appear to be longlasting and implicate multiple brain circuits (Volkow and $\mathrm{Li}, 2005$ ). New behavioral and pharmacological treatments need to be developed to treat such neuroadaptations. However, the development of new pharmacological interventions requires understanding the effects of potential medications in a neural system. Because small animals have been widely used as laboratory models to study neuroadaptations and pharmacological interventions, there are clear needs for sensitive methods to globally map the effects of pharmacological actions on central nervous system in small animals. The imaging methods used to detect the brain functional changes in small animal models for drug abuse/addiction include fMRI and PET, these techniques associate with either high cost or using ionizing radiations.

High-resolution optical imaging also has been widely applied in small animal studies of basic brain function and diseases. However, due to strong light scattering in soft tissue, highresolution, purely-optical imaging modalities usually only have an imaging depth of $\sim 1 \mathrm{~mm}$

\footnotetext{
(C) 2010 Elsevier B.V. All rights reserved.
}

Publisher's Disclaimer: This is a PDF file of an unedited manuscript that has been accepted for publication. As a service to our customers we are providing this early version of the manuscript. The manuscript will undergo copyediting, typesetting, and review of the resulting proof before it is published in its final citable form. Please note that during the production process errors may be discovered which could affect the content, and all legal disclaimers that apply to the journal pertain. 
in soft tissue. As a result, the application of high-resolution, purely-optical imaging for small animal brain studies often requires removing or thinning the skull, which are invasive procedures associated with significant risks and draw-backs.

Photoacoustic tomography (PAT) (also refered as thermoacoustic (Kruger et al., 1999), or optoacousitc tomography (Esenaliev et al., 1999)) overcomes the limitations of other existing optical imaging modalities and combines optical contrast with ultrasonic resolution. PAT is based on the generation of photoacoustic waves by safely depositing short-pulsed optical energy into tissue. Each laser pulse causes a rapid temperature rise on the order of 10 millidegrees. The ultrasonic emission due to thermoelastic expansion can be detected with a single-element ultrasonic transducer or an array of ultrasonic transducers and then used to reconstruct an image (Wang, 2008). In PAT, the task is to determine the relative optical absorption density (or specific absorption) distribution from the measured photoacoustic data, i.e., to map the optical absorption heterogeneity of the tissue. PAT can provide images with optical contrast and ultrasound resolution in regions up to $5 \mathrm{~cm}$ deep (Ku and Wang, 2005).

PAT has been used in in vivo small animal experiments for noninvasive monitoring of angiogenesis, blood oxygenation, and functional changes in the brain (Li and Wang, 2009; Wang et al., 2006). In this paper, for the first time to the authors' knowledge, PAT is applied to detect the activation of the brain function induced by using of cocaine in small animals. Brain activation due to cocaine abuse in small animals has been one of popular models to study drug abuse/addiction, and cocaine is one of most commonly use addictive drugs. With repeated use, cocaine can lead to addiction. Cocaine administration produced a dosedependent neuronal activation that is accompanied by changes in brain metabolic rate. More specifically, acute cocaine administration will affect the level of dopamine in the brain, and as a result, changes in cerebral blood volume will be induced. Many studies in the past have demonstrated regionally restricted activation in the brain of small animals after acute cocaine administration with fMRI. PAT, with all the merits aforementioned, has the potential to be an alternative imaging modality for this application, and provide complementing information to fMRI and PET.

In this study, we use PAT to monitor brain functional activation by monitoring the changes of the blood volume at the cerebral cortex surface of small animals. In blood, PA signal is mainly determined by the concentration of hemoglobin. During the performance of PAT, we used $797 \mathrm{~nm}$ optical wavelength light, which is an isospectral point, i.e., the wavelength that the optical absorption levels of oxygenated hemoglobin $\left(\mathrm{HbO}_{2}\right)$ and de-oxygenated hemoglobin $(\mathrm{Hb})$ are the same, and therefore, the obtained photoacoustic signal amplitude are proportional to the local blood volume (Wang et al., 2003; Wang et al., 2006). As a result, the changes in blood volume can be detected by monitoring the amplitude changes of the detected photoacoustic signals. On a PAT image, the changes in photoacoustic signal amplitude will result in the changes in image contrast.

\section{Materials and Methods}

\subsection{Photoacoustic tomography system}

We used a photoacoustic tomography (PAT) system, and the schematic of the system is shown in Fig. S1 in Supplementary. The PAT system has a tunable OPO laser (Surelite OPO PLUS; Continuum, Santa Clara, CA) pumped by an Nd:YAG laser (Surelite III; Continuum, Santa Clara, CA) that provided $10 \mathrm{~Hz}$ laser pulses with $6 \mathrm{~ns}$ pulse width. The laser beam was expanded and homogenized by a diffuser lens (grounded glass). The incident energy density of the laser that reached the surface of the rat head was less than $20 \mathrm{~mJ} / \mathrm{cm}^{2}$, which complied the safety limit for human skin exposure (Laser Institute of America, 2000). We used 797 
nm wavelength of laser to illuminate shaved rat head, which emits ultrasound due to photoacoustic effect. The generated photoacoustic signals from the rat's brain were detected by 5-MHz unfocused ultrasonic transducers (V310, Olympus NDT, Waltham, MA) which were scanned around the rat's head horizontally (Ku et al., 2004; Wang et al., 2003). Two transducers were used during data acquisition. The two transducers were set apart by $180^{\circ}$, and carefully aligned to face the scanning center. As a result, each transducer only needed to scan $180^{\circ}$ to get a full circular scan. The detected signals were amplified by a multi-channel pre-amplifier, collected through a multiple-channel data acquisition board (Gage, Lockport, IL), and then downloaded to a personal computer for post-analysis. During data collection, a stepper motor was controlled to rotate two ultrasonic transducers. The scanning step was 2 degrees with 20-time average. Data collected through the two ultrasonic transducers was combined and compensated by laser power level that is measured by photo diode for imaging reconstruction. The system was able to acquire one PAT image every five minutes. In order to monitor the changes in the rat brain continuously, PAT system had to continuously run to obtain the data periodically.

\subsection{Animal preparation}

Sprague Dawley rats were used as our animal model. For imaging procedures, animals were initially anesthetized with a mixture of ketamine $(87 \mathrm{mg} / \mathrm{kg}$ body weight $)$ and xylazine $(13 \mathrm{mg} / \mathrm{kg}$ body weight), the subsequent anesthesia (about one hour after the initial anesthesia) was maintained with the inhalation of 1.0 2.0\% isoflurane mixing with oxygen. The hairs on the head of animal were shaved with an electric shaver first, and then the hair removal lotion were applied to depilate the rest of hairs. At the end, the lotion was wiped away with gauzes and followed by washing with warm water. The procedure was repeated until the hairs were completely removed. At this point, the animals were kept under anesthesia with a gas anesthesia machine during the balance of the experiment. Surgical tapes were used to gently secure the animal onto a warmed pad and secured in the imaging aperture in which only light pressure was needed. A custom-designed surgical stereotaxic frame was also used to fix the head of the animal with ear-pins and a tooth-pin. The body temperature was maintained with a heating lamp. The heartbeat of the animal remained about $250 \pm 10$ per minute.

\section{3 imaging process}

For the in vivo animal experiment, fifteen Sprague-Dawley rats (140 g to $220 \mathrm{~g}$ body weight) were used, and the details for animal preparation are also presented in supplementary information. These rats were divided into 3 groups and injected cocaine hydrochloride in $0.9 \%$ sterile saline at given dose of 0 (sham treatment, saline only), 2.5, and $5.0 \mathrm{mg} / \mathrm{kg}$ body weight respectively. Totally, each animal was given $0.9 \%$ sterile saline at a dose of $1 \mathrm{ml} / \mathrm{kg}$ body weight. About two hours after administering the initial anesthesia, the cocaine hydrochloride in sterile saline solution was injected through the rat tail veins. During the experiment, the heart rate and blood oxygenation was monitored with a pulseoximeter (Nonin medical, PulseSense VET, Plymouth, MN). An initial PAT image was obtained right before the injection of cocaine hydrochloride solution. This image would serve as the reference image. Then the drug was injected and PAT was performed continuously for a total period of at least 60 minutes.

\section{Results}

As the first step, we tested PA signals generated from cocaine hydrochloride solution at 797 $\mathrm{nm}$. The concentration of cocaine in saline solution is $1.65 \mathrm{e}-5 \mathrm{~mol} / \mathrm{ml}$ that has the similar concentration with the case of $5 \mathrm{mg} / \mathrm{kg}$ (body weight) of cocaine. The signal from the 
solution was indistinguishable from noise, and therefore, the optical absorption of cocaine hydrochloride solution is negligible.

The example of obtained PAT image of brain cortex surface is shown in Fig. 1. Fig. 1a and $1 \mathrm{~b}$ were taken before and 60 minutes after the injection of $5 \mathrm{mg} / \mathrm{kg}$ cocaine hydrochloride, respectively. Compared with Fig. 1a, Fig. $1 \mathrm{~b}$ reveals the brain vasculature with greater clarity. This enhanced clarity is the result of the brain function activation due to the injection of cocaine hydrochloride, which stimulates the central nervous system and causes the increase of blood flowing into the brain. Consequently, the amplitudes of the detected photoacoustic signals will increase, and hence the contrast between blood in vessels and the background brain tissues will be enhanced. A differential image shown in Fig. 1c was obtained by subtracting the amplitude of the pre-injection data (Fig. 1a) from that of the post-injection data (Fig. 1b) and reconstructing. This image displays the distribution of differential optical absorption (i.e., the changes in blood volume) in the rat brain as induced by cocaine hydrochloride. All the PAT images of the rat brain match well with the openskull anatomical photograph in Fig. 1d.

The changes in the amplitudes of photoacoustic signals after cocaine hydrochloride injection were calculated for all PAT images. The signals were integrated over each image and then normalized to the signal integration of the reference image. The signal increases are presented in percentage, with $0 \%$ indicates no increase comparing with the reference signal. Fig. 2 shows the results of the changes in photoacoustic signal amplitudes averaged on brain cortex after the injection of three concentrations $(0 \mathrm{mg} \cdot \mathrm{kg}, 2.5 \mathrm{mg} / \mathrm{kg}$, and $5 \mathrm{mg} / \mathrm{kg})$ of cocaine hydrochloride. We notice that after the injection of sterile saline solution $(1 \mathrm{ml} / \mathrm{kg}$ body weight) without cocaine hydrochloride, the amplitudes of photoacoustic signals decline, and stay at a constant level for up to 60 minute before it starts to come back to the original level. This could be due to physiological reaction as the decreases of the hemoglobin concentration under isoflurane anesthesia. This result will serve as the base line reference for the estimation of the brain response solely due to the injection of cocaine hydrochloride. The results from the injection of $2.5 \mathrm{mg} / \mathrm{kg}$ cocaine hydrochloride shows the decrease on the amplitude of PA signals right after the injection, and the amplitude of PA signals recovers after about 10 minutes. Since PA signal amplitude is related to the blood volume, therefore, this result indicates a decrease in blood volume on brain cortex right after the injection of cocaine hydrochloride, and a recovery in blood volume 10 minutes later. The initial decrease in blood volume on the brain cortex is consistent with the reported result that acute cocaine injection may decrease the cerebral blood volume (Luo et al., 2009). For 5 $\mathrm{mg} / \mathrm{kg}$ cocaine hydrochloride injection, however, and we did not observe the initial decrease in the PA signal amplitude, instead, the PA signal amplitude increases after the injection. This result may be due to the high concentration of cocaine hydrochloride we used. The error bars showed in Fig. 2 are the standard deviations of the measurements from five animals.

\section{Discussion and conclusions}

This research showed PAT is feasible for the study of brain activation due to drug abuse. The major advantage of the PAT brain imaging is the combination of optical contrast and ultrasound resolution in deep regions. The ability of providing non-invasive brain functional imaging by PAT offers an important tool for small animal research. PAT has also demonstrated the ability of imaging through a much thicker monkey brain (Yang and Wang, 2008). Therefore, this technique may also be used to perform human brain imaging, or at least infant brain imaging. 
Although current study only used $797 \mathrm{~nm}$ wavelength light to measure the changes in blood volume (total hemoglobin), PAT technique can be used to measure $\mathrm{HbO}_{2}$ and $\mathrm{Hb}$ separately when two or more optical wavelengths are used. The separated measurements on $\mathrm{HbO}_{2}$ and $\mathrm{Hb}$ will shed more lights on the brain function activation detection.

Images from our current PAT system produced good cerebral cortex surface images. However, due to the poor resolution on the direction perpendicular to the cortex surface, current system cannot resolve between signals generated from the inside of the brain and signals generated from the cortex surface. A reflection-mode photoacoustic imaging system could be more suitable to image inside of the brain (Song and Wang, 2007).

In summary, PAT was used to detect effects of addicted stimulus due to the injection of cocaine hydrochloride. Stimulation of cocaine induces brain function activation, which results in the crease of total blood volume in the brain. On PAT images, the change of blood volume was detected by monitoring the image contrast enhancement. Our results show that PAT technique can non-invasively detect the brain function activation induced by drug abuse through detecting the blood volume change in small animals.

\section{Supplementary Material}

Refer to Web version on PubMed Central for supplementary material.

\section{References}

Andlin-Sobocki P, Rehm J. Cost of addiction in Europe. European Journal of Neurology. 2005; 12:2833. [PubMed: 15877775]

Esenaliev RO, Karabutov AA, Oraevsky AA. Sensitivity of laser opto-acoustic imaging in detection of small deeply embedded tumors. IEEE Journal of Selected Topics in Quantum Electronics. 1999; 5:981-8.

Grant BF, Dawson DA. Age of onset of drug use and its association with DSM-IV drug abuse and dependence: Results from the National Longitudinal Alcohol Epidemiologic Survey. Journal of Substance Abuse. 1998; 10:163-73. [PubMed: 9854701]

Kruger RA, Reinecke DR, Kruger GA. Thermoacoustic computed tomography-technical considerations. Medical Physics. 1999; 26:1832-7. [PubMed: 10505871]

Ku G, Wang LHV. Deeply penetrating photoacoustic tomography in biological tissues enhanced with an optical contrast agent. Optics Letters. 2005; 30:507-9. [PubMed: 15789718]

Ku G, Wang X, Stoica G, Wang LV. Multiple-bandwidth photoacoustic tomography. Phys Med Biol. 2004; 49:1329-38. [PubMed: 15128208]

Laser Institute of America. American National Standard for Safe Use of Lasers ANSI Z136.1-2000. American National Standards Institure I. , editor. 2000.

Li CH, Wang LHV. Photoacoustic tomography and sensing in biomedicine. Physics in Medicine and Biology. 2009; 54:R59-R97. [PubMed: 19724102]

Luo F, Schmidt KF, Fox GB, Ferris CF. Differential responses in CBF and CBV to cocaine as measured by fMRI: Implications for pharmacological MRI signals derived oxygen metabolism assessment. J Psychiatr Res. 2009; 43:1018-24. [PubMed: 19135215]

Song KH, Wang LV. Deep reflection-mode photoacoustic imaging of biological tissue. Journal of Biomedical Optics. 2007; 12 Article No. 060503.

Volkow N, Li TK. The neuroscience of addiction. Nature Neuroscience. 2005; 8:1429-30.

Wang LV. Tutorial on photoacoustic microscopy and computed tomography. IEEE J Sel Top Quant. 2008; 14:171-9.

Wang X, Pang Y, Ku G, Xie X, Stoica G, Wang LV. Noninvasive laser-induced photoacoustic tomography for structural and functional in vivo imaging of the brain. Nat Biotechnol. 2003; 21:803-6. [PubMed: 12808463] 
Wang X, Xie X, Ku G, Wang LV, Stoica G. Noninvasive imaging of hemoglobin concentration and oxygenation in the rat brain using high-resolution photoacoustic tomography. J Biomed Opt. 2006; 11:024015. [PubMed: 16674205]

Yang XM, Wang LV. Monkey brain cortex imaging by photoacoustic tomography. Journal of Biomedical Optics. 2008; 13:044009. [PubMed: 19021337] 


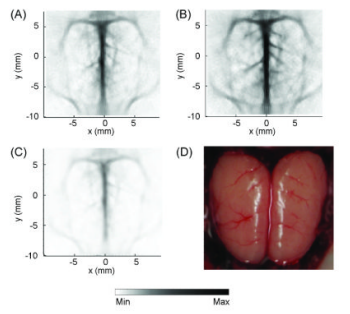

Fig. 1.

Non-invasive PAT imaging of a rat's cerebral cortex (A) before the injection of cocaine hydrochloride; (B) $60 \mathrm{~min}$ after the injection of $5 \mathrm{mg} / \mathrm{kg}$ concentration cocaine hydrochloride. (C) A pixel-wise differential image (image B - image A). (D) An open-skull photograph of the rat's cerebral cortex. 


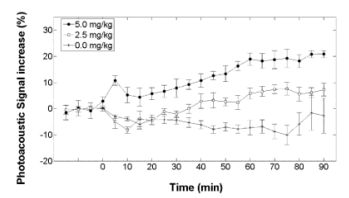

Fig. 2.

Averaged photoacoustic signal amplitude increases at cerebral cortex of rats. Three concentrations of cocaine hydrochloride are used: 0 (pure saline solution), 2.5 , and $5.0 \mathrm{mg} /$ $\mathrm{kg}$ body weight. The error bars are standard deviations from the average of the five animals in each group. 